\title{
Synthesis of Cosecant Linear Antenna Array Pattern Using a Novel Modified Invasive Weeds Optimization
}

\author{
El Hadi Kenane ${ }^{1,2}$, Farid Djahli ${ }^{1}$, Arres Bartil ${ }^{1}$ \\ ${ }^{1}$ LIS Laboratory, Institute of Electronics, University of Setif 1, \\ 19000 Setif, Algeria \\ ${ }^{2}$ LGE Laboratory, Department of Electronics Engineering, University of M'sila, \\ Ichbilia St. 28000 M'sila, Algeria \\ kenaneh@yahoo.fr
}

\begin{abstract}
${ }^{1}$ Abstract-In this paper, a new modified method is presented for the synthesis of a linear antenna array to obtain a desired array pattern (shaped or pencil) with low side lobe level. Based on the original invasive weeds optimization (IWO), our modified IWO (MIWO) uses the process of mutation for the calculation of standard deviation. The shaped beam pattern is synthesized by respecting a desired cosecant pattern and supressing side lobe level to $-\mathbf{2 5} \mathbf{~ d B}$. To achieve the desired pattern, both amplitude and phase of each element in the array are optimized when the spacing between the elements is fixed to the half wavelength. For the desired pencil beam pattern, the inter-element spacing's are controlled while maintaining uniform excitations. Selected examples, for both shaped and pencil beam, are presented to show the effectiveness and flexibility of the proposed method.
\end{abstract}

Index Terms-Antenna array, cosecant array pattern, invasive weeds optimization, side lobe level, antenna synthesis.

\section{INTRODUCTION}

The recent developments in wireless telecommunications technologies require networks with enhanced characteristics like capacity and coverage. Whatever its complexity, a single antenna element is not able to fit with the requests of environment, but these limitations could be overcome by the synthesis of antenna arrays. In wireless mobile telecommunications, the most commonly used topology is the uniform linear array [1]. Moreover, in the past decades, many researches have been performed in the field of linear array synthesis. The goal is to fit with new requirements in radiation characteristics by altering current distribution (amplitude and/or phase). The principal requirements to be satisfied are minimum side lobe level (SLL) [2], nulls towards interferers [3], shaped beam [4]-[6].

The synthesis of antenna array that gives a desired pattern by controlling amplitude and phase is a nonlinear problem. Since the classical synthesis methods often fall in local optima, many stochastic methods have been used for solving the array design problems. Among the recent and robust algorithms which have been successfully employed, these methods include: genetic algorithm (GA) [7], ant colony optimization (ACO) [8], particle swarm optimization (PSO) [9], flocks of starling (FSO) [10], cat swarm optimization (CSO) [11], artificial bees colony (ABC) [12] and plant growth simulation algorithm (PGSA) [13].

On the other hand, in [14], Mehrabian and Lucas proposed a new algorithm based on the ecological behaviour of colonizing weeds, known as invasive weeds optimization (IWO). In the last few years, IWO became popular in the field of electromagnetic problems due to its robustness and flexibility [15].

In this paper, a new modified version of IWO, known as MIWO, is presented for the synthesis of linear array antenna to obtain an array pattern with shaped beam. All the steps in our modified IWO are the same in the original IWO except the reproduction process. The procedure of reproduction is based on a new dispersal technique as follow. For each iteration index, a random number is generated and compared to a fixed number called the probability of mutation $\left(P_{m}\right)$. If the generated number is greater than $P_{m}$, the algorithm will replace the value of the calculated standard deviation $S D$ of the current iteration by its initial value. This process of mutation allows a larger dispersion of seeds around the parent plant, thus avoiding getting stuck in local minima. To illustrate the performance of the proposed algorithm, MIWO is applied to synthesis of both pencil beam patterns and shaped beam patterns.

\section{ANTENNA ARRAY PROBLEM FORMULATION}

Let us assume that a $2 \mathrm{~N}$-element linear array is symmetric with respect to its center, placed along $x$ axis as shown in Fig. 1. Mathematically, the Array Factor $(A F)$ can be written as

$$
A F(\theta)=2 \sum_{n=1}^{N} a_{n} \cos \left(\frac{2 \pi}{\lambda} x_{n} \sin \theta+\varphi_{n}\right)
$$


axis of the array, $a_{n}$ and $\varphi_{n}$ represent the amplitude and phase weights of the $n^{\text {th }}$ element on each side of the array, respectively, $x_{\mathrm{n}}$ is the position of the $n^{\text {th }}$ element in the array.

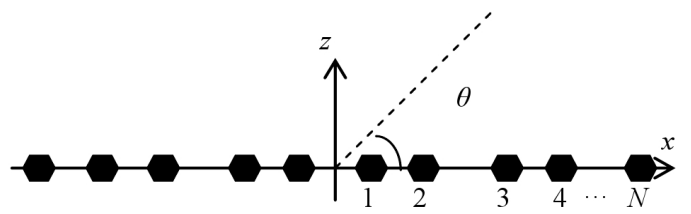

Fig. 1. The geometry of $2 N$ element symmetric linear array of isotropic sources positioned along $\mathrm{x}$ axis.

In our study, by varying both amplitude and phase of each element in the array, the characteristics of the array factor will be controlled during the synthesis process. To overcome the effect of mutual coupling, an allowed distance of interelements is fixed to $0.5 \lambda$.

In order to synthesize the array desired pattern, both in the cosecant region and in the predetermined side-lobe regions, a cost function for these requirements is given by [16]:

$$
\begin{gathered}
C F_{1}=w_{1} \sum_{\theta=0^{\circ}}^{180^{\circ}}\left[A F(\theta)-S_{\max }(\theta)\right] \times \\
\times\left[\frac{1+\operatorname{sgn}\left(A F(\theta)-S_{\max }(\theta)\right)}{2}\right]+ \\
+w_{2} \sum_{\theta=0^{\circ}}^{180^{\circ}}\left[S_{\min }(\theta)-A F(\theta)\right] \times \\
\times\left[\frac{1+\operatorname{sgn}\left(S_{\min }(\theta)-A F(\theta)\right)}{2}\right],
\end{gathered}
$$

where $w_{1}$ and $w_{2}$ are the weights of the cost function, generally both equal to 1 . $\left(w_{1}=w_{2}=1\right)$,

$S_{\min }(\theta)=\left\{\begin{array}{l}\frac{[\csc (\cos (\theta)) \cdot \csc (\cos (99))]}{M_{1}}, 95.8^{\circ} \leq \theta \leq 120^{\circ}, \\ 10^{-\frac{70}{20}}, \text { elsewhere, }\end{array}\right.$

here $M_{1}=1.122[\csc (\cos (95.8)) \times \csc (\cos (99))]$,

$$
S_{\max }(\theta)=\left\{\begin{array}{l}
1,90^{\circ} \leq \theta<97^{\circ} \\
\frac{\csc (\cos \theta) \times \csc \left(\cos 99^{\circ}\right)}{\mathrm{M}_{2}}, 97^{\circ} \leq \theta<120^{\circ}, \\
\frac{\csc \left(\cos 120^{\circ}\right) \times \csc \left(\cos 99^{\circ}\right)}{\mathrm{M}_{2}}, 120^{\circ} \leq \theta<127^{\circ}, \\
10^{-\frac{25}{20}}, \text { elsewhere }
\end{array}\right.
$$

here $M_{2}=\csc \left(\cos \left(97^{\circ}\right)\right) \times \csc \left(\cos \left(99^{\circ}\right)\right)$.

As it can be seen, $S_{\max }$ allows a tolerance of $1 \mathrm{~dB}(1.122$ in linear scale, as was extracted from the expression of $M_{1}$ ) with $S_{\min }$ between $95.8^{\circ}$ and $120^{\circ}$. Here $S_{\max }$ and $S_{\min }$ represent the maximum and minimum shaping region, respectively. A desired pattern must be situated inside the shaping region. To achieve this goal, our MIWO method is used.

\section{ModifIED INVASIVE WEEDS OPTIMIZATION}

IWO is a recent method, compared to genetic algorithm (GA) and other traditional stochastic methods, based on the colonizing behavior of the invasive weeds in the nature. Plants invade cropping field, occupy the free space around this field and grow to get new weeds, and so on. Starting from random positions of a non-uniform linear array, the optimum positions of the elements will be found by suppressing side lobes in both regions outside the main beam, with symmetric nulls in some directions. In the proposed MIWO, a mutation process in the calculation of $S D$ is added; this allows a larger dispersion of seeds around the parent plant. Hence, new weeds will then grow randomly. Their number is related to the fitness value of their parents. The algorithm of MIWO method is organized as follows.

\section{A. Initialization}

First, a finite number of plants are randomly spread over search space ( $N$ dimensions). This initial population is denoted as $P O P=\left[P_{1}, P_{2} \ldots, P_{\text {pop_ini }}\right]$ where $p o p \_i n i$ is the number of generated plants. Each plant is considered as a proposed solution in the search space and termed as $P_{i}=\left[I_{1}\right.$, $\left.I_{2}, \ldots, I_{N}\right]$, where $I_{n}$ represent both amplitude and phase of the $n^{\text {th }}$ element on each side of the array.

\section{B. Evaluation}

The fitness reflects an evaluation of how good the plant is. The optimal plant (vector solution) is the one which minimizes the cost function defined by the designer.

\section{Reproduction}

Depending on its fitness value, a plant can produce a number of seeds from 0 to a fixed number. The best plant in the colony will produce a maximum number of seeds, while the worst plant cannot produce any seed. Between these extremely plants fitness, the number of produced seeds is given by

$$
N s\left(P_{i}\right)=\text { integer }[N b r]
$$

where

$$
N b r=\mathrm{m}_{\mathrm{s}}+\left(\frac{M s-m_{s}}{B C-W C}\right)\left[C\left(P_{i}\right)-W C\right],
$$

where $M s$ and $m_{s}$ are the maximum and minimum numbers of generated seeds, respectively. $B C$ and $W C$ are the best and worst cost in the actual population, respectively. $C\left(P_{i}\right)$ is the cost function of $i^{\text {th }}$ plant in the population and $[\mathrm{Nbr}]$ is a mathematical function that gives the greatest integer of $\mathrm{Nbr}$.

Next, the produced seeds will be spread around its parent with a random distance using uniform distribution with the mean equal to 0 and a standard deviation $S D$ that will decrease iteratively following the equation below

$$
S D_{i t e r}=\frac{\left(i t r_{\max }-i t r\right)}{\left(i t r_{\max }\right)^{\text {mod }}}\left(S D_{i n i}-S D_{f n l}\right)+S D_{f n l} \text {, }
$$

where, $i t r_{\max }$ is the maximum number of iterations and itr is the actual iteration index. $S D_{\text {ini }}$ and $S D_{\text {fnl }}$ present the initial and final values of standard deviation, respectively; mod is 
the modulation index, usually equal to 3 .

\section{Mutation}

In IWO, many works investigate on the choice of standard deviation value, as the most important parameter in this optimization method. A good parameter choice is essential for a fast convergence of the process. Many works have been done to achieve its optimal value. For instance, in [17], each weed in the population has its own standard deviation value depending to its cost function in the actual population; whereas a periodical variation can be added to the standard deviation.

In the present study, the idea is inspired from the mutation process of the genetic algorithms (GA) [7]. First, a probability $P_{\mathrm{m}}$ is fixed. If $P_{\mathrm{m}}$ is lower than a randomly generated value in the range $[0,1]$, then the standard deviation value of the current iteration $S D_{\text {iter }}$ will be replaced by its initial value $S D_{\text {ini }}$, otherwise, the standard deviation $S D_{\text {iter }}$ will be calculated as indicated in (7). The steps of the proposed algorithm are as follows:

Step 1: A random number is generated.

Step 2: A fixed probability of mutation $P_{\mathrm{m}}$ is chosen.

Step 3: If the random number is greater than $P_{\mathrm{m}}$ then $S D_{\text {iter }}=S D_{\text {ini }}$;

Else $S D_{\text {iter }}$ is calculated from (7).

Step 4: Repeat this algorithm for each iteration.

\section{E. Limitation}

The seeds will grow and become new weeds. These weeds will be added to the colony till a maximum number of plants pop_max is reached. In this case, a competitive exclusion will begin to keep only the best pop_max ${ }^{\text {th }}$ plants in the colony, and discard the rest.

\section{F. Stop Criteria}

Generally, a maximum number of iterations is taken as a stop criterion.

\section{NUMERICAL RESULTS}

In order to assess the performance of the proposed MIWO for the synthesis of linear arrays, two examples have been chosen and presented. Each example has $2 N$ number of elements. All algorithms have been run 50 times. The best results are compared. A comparison is presented between the proposed method and other instantiations in the literature such as ABC [12], PSO [9] and ACO [8]. In this study, the initial population is fixed to $4 N$ and the maximum number of population is fixed to twice of initial population. The standard deviations $S D_{\text {ini }}$ and $S D_{\text {fnl }}$ are set to 0.05 and $10^{-7}$, respectively. The iteration number is fixed to 100 and $P_{m}$ is fixed to 0.5 and will be decreased iteratively.

In the first example, a shaped beam pattern synthesis of $2 N=24$ elements has been examined by controlling the amplitude and phase of each element on each side of the array. The inter-element spacing is considered equal to $0.5 \lambda$. In Fig. 2, the cosecant pattern is obtained using MIWO by determining both amplitude and phase of the array elements, when the optimal values are tabulated in Table I.

In this case, as in [16], a reduction of $20 \%$ in the number of array elements is obtained in comparison with the same goal achieved for 30 elements using tabu search algorithm
(TSA) [4].

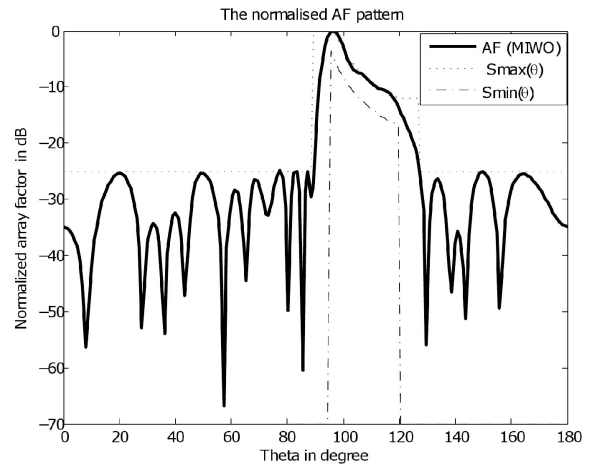

Fig. 2. The optimized array factor obtained using MIWO for 24 elements (solid line) and the mask of desired pattern (dashed line), where the dotdashed line represents $S_{\min }$ and the dotted ( ) represents $S_{\max }$.

TABLE I. OPTIMIZED ELEMENT EXCITATIONS (AMPLITUDE AND PHASE) OBTAINED USING MIWO FOR THE ARRAY FACTOR SKETCHED IN FIG. 1.

\begin{tabular}{|c|c|c|}
\hline Element number $(\boldsymbol{n})$ & $\begin{array}{c}\text { Normalized amplitude } \\
\text { weights }\left(\boldsymbol{a}_{\boldsymbol{n}}\right)\end{array}$ & $\begin{array}{c}\text { Static phase weights } \\
\left(\boldsymbol{\varphi}_{\boldsymbol{n}}\right)\end{array}$ \\
\hline 1 & 0.9835 & 20.7204 \\
\hline 2 & 0.8638 & 57.5860 \\
\hline 3 & 0.5365 & 86.3196 \\
\hline 4 & 0.3402 & 91.3742 \\
\hline 5 & 0.3137 & 87.1511 \\
\hline 6 & 0.2787 & 124.6700 \\
\hline 7 & 0.2753 & 138.9213 \\
\hline 8 & 0.2006 & 143.9706 \\
\hline 9 & 0.1441 & 148.9622 \\
\hline 10 & 0.1890 & 179.9825 \\
\hline 11 & 0.0753 & 151.3751 \\
\hline 12 & 0.1124 & 179.9522 \\
\hline
\end{tabular}

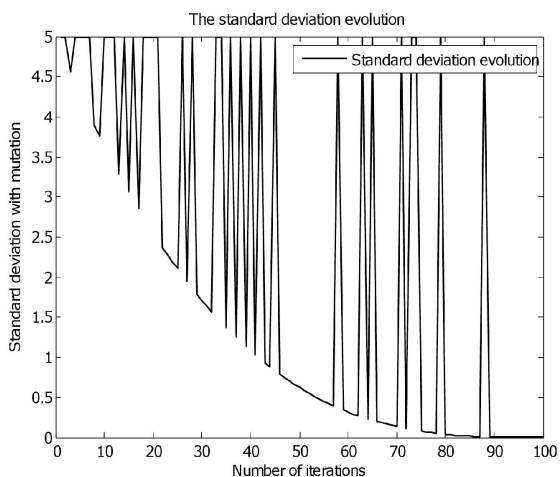

Fig. 3. Evolution of the standard deviation values versus number of iterations for the 24 element array which present 32 mutations.

In the same way, the problem dimension $(N=12)$ is reduced to the half when the symmetry in controlling parameters is proposed. The dynamic range ratio $(D R R)$ of amplitude is acceptable (equal to 13.06), in comparison with the very high value $(D R R=171.65)$ found by [6] and 15.26 found by [4]. Fig. 3 shows the evolution of standard deviation values versus the number of iterations, when more than 32 mutations are shown. Causing to the iterative decrease of $P_{m}$, it can be seen that most mutations are found in the first half range of iterations.

\section{COMPARATIVE STUdY}

In order to evaluate the capabilities of the proposed method, a good instantiation of the design problem is solved using our MIWO, ACO [8], PSO [9], and CLPSO [18]. A non-uniform linear array of $2 N=10$ elements is considered with uniform excitation $\left(a_{n}=1\right.$ and $\left.\varphi_{n}=0\right)$. By controlling 
the inter-elements spacing, an optimized array pattern should present a minimum $S L L$ with a beam-width equal or less to that of the corresponding uniform array. The number of iterations is fixed to 1000 , and the cost function is given by [9]

$$
C F 2=\max _{\theta \in S}\left\{A F_{\mathrm{dB}}(\theta)\right\}+\Xi \cdot \max \left\{0,\left|B W_{\mathrm{c}}-B W_{\mathrm{d}}\right|-1\right\},
$$

where $S$ is the space spanned by the angle $\theta$ excluding the main beam, $B W_{\mathrm{c}}$ and $B W_{\mathrm{d}}$ denote the calculated and desired beamwidth, respectively and $\Xi$ is a very large number.

\section{TABLE II. OPTIMIZED ELEMENT POSITIONS OF 10 ELEMENT LINEAR ARRAY USING MANY METHODS IN TERM OF} WAVELENGTH.

\begin{tabular}{|c|c|c|c|c|c|}
\hline $\mathbf{n}$ & uniform & MIWO & ACO [8] & CLPSO [18] & PSO [9] \\
\hline 1 & 0.2500 & 0.2290 & 0.2500 & 0.2515 & 0.2515 \\
\hline 2 & 0.7500 & 0.7352 & 0.5500 & 0.7110 & 0.5550 \\
\hline 3 & 1.2500 & 1.2490 & 1.0500 & 1.2080 & 1.0650 \\
\hline 4 & 1.7500 & 1.8973 & 1.5500 & 1.8350 & 1.5000 \\
\hline 5 & 2.2500 & 2.6450 & 2.1500 & 2.5585 & 2.1100 \\
\hline
\end{tabular}

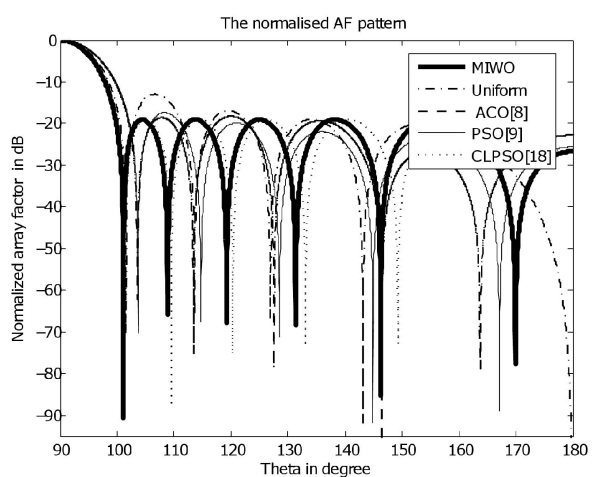

Fig. 4. Comparison of normalized radiation patterns of 10 element linear array obtained by different stochastic methods versus our proposed method.

The optimal positions are listed in Table II, when the corresponding radiation pattern is shown in Fig. 4. As it can be seen, the best design is obtained by MIWO when a minimum SLL is lower than $-19.06 \mathrm{~dB}$ with a beamwidth close to that of the uniform array.

\section{CONCLUSIONS}

In this paper, a new algorithm denoted as modified invasive weeds optimization (MIWO) is introduced for the synthesis of both cosecant array pattern and pencil beam pattern of linear array. MIWO has been compared to other methods when the different results show the robustness and the performance of the proposed method. Moreover, the use of a mutation in the standard deviation calculation allows the search space to be better explored avoiding falling in local minimum. In consequence, the convergence will be accelerated. Many other extensions in array design could be also easy for implementation. Because of the good performance of MIWO, it can be used as an alternative optimization method to solve many problems in antenna design.

\section{REFERENCES}

[1] C. Godara, "Applications of antenna arrays to mobile communications, part I: performance improvement, feasibility, and system considerations", in Proc. the IEEE, vol. 85, no. 7, 1997, pp. 1031-1060. [Online] Available: http://dx.doi.org/10.1109/5.611108

[2] K. N. Abdul Rani, M. F. Abdul Malek, N. Siew-Chin, "Natureinspired Cuckoo search algorithm for side lobe suppression in a symmetric linear antenna array", Radio Engineering, vol. 21, no. 3, pp. 865-874, 2012.

[3] N. G. Gomez, J. J. Rodriguez, K. L. Melde, K. M. McNeill, "Design of low-side lobe linear arrays with high aperture efficiency and interference nulls", IEEE Antennas and Wireless Propagation Letters, vol. 8, pp. 607-610, 2009. [Online] Available: http://dx.doi.org/10.1109/lawp.2009.2018126

[4] A. Akdagli, K. Guney, "Shaped-beam pattern synthesis of equally and unequally spaced linear antenna arrays using a modified tabu search algorithm", Microwave And Optical Technology Letters, vol. 36, no. 1, pp. 16-20, 2003. [Online] Available: http://dx.doi.org/10.1002/ mop. 10657

[5] J. A. R. Azevedo, "Shaped beam pattern synthesis with non-uniform sample phases", Progress In Electromagnetics Research B, vol. 5, pp. 77-90, 2008. [Online] Available: http://dx.doi.org/10.2528/ PIERB08020103

[6] A. K. Behera, A. Ahmad, S. K. Mandal, G. K. Mahanti, R. Ghatak, "Synthesis of cosecant squared pattern in linear antenna arrays using differential evolution", in Proc. IEEE Conf. Information and Communication Technologies, pp. 1025-1028, 2013. [Online] Available: http://dx.doi.org/10.1109/CICT.2013.6558248

[7] R. L. Haupt, "Phase-only adaptive nulling with a genetic algorithm", IEEE Trans. Antennas and Propagation, vol. 45, no. 6, pp. 10091015, 1997. [Online] Available: http://dx.doi.org/10.1109/8.585749

[8] E. R. Iglesias, O. Q. Teruel, "Linear array synthesis using an ant colony optimization based algorithm", IEEE Antennas and propagation Magazine, vol. 49, no. 2, pp. 70-79, 2007. [Online] Available: http://dx.doi.org/10.1109/MAP.2007.376644

[9] M. Khodier, C. G. Christodoulou, "Linear array geometry synthesis with minimum side lobe level and null control using particle swarm optimization", IEEE Trans. antennas and propagation, vol. 53, no. 8 , 2005, pp. 2674-2679. [Online] Available: http://dx.doi.org/10.1109/ TAP.2005.851762

[10] F. Riganti Fulginei, A. Salvini, "Hysteresis model identification by the flock-of-starlings optimization", International Journal of Applied Electromagnetics and Mechanics, vol. 30, no. 3-4, pp. 321-331, 2009. [Online] Available: http://dx.doi.org/10.3233/JAE-2009-1032

[11] L. Pappula, D. Ghosh, "Linear antenna array synthesis using cat swarm optimization", International journal of electronics and communications $A E U$, vol. 68, no. 6, pp. 540-549, 2014. [Online] Available: http://dx.doi.org/10.1016/j.aeue.2013.12.012

[12] B. Basu, G. K. Mahanti, "Fire fly and artificial bees colony algorithm for synthesis of scanned and broadside linear array antenna", Prog Electromagn Res B, vol. 32, pp. 169-190, 2011. [Online] Available: http://dx.doi.org/10.2528/PIERB11053108

[13] C. Tang, R. Liu, J. Ni, "A novel wireless sensor network localization approach: localization based on plant growth simulation algorithm", Elektronika ir Elektrotechnika, vol. 19, no. 8, pp. 97-100, 2013. [Online] Available: http://dx.doi.org/10.5755/j01.eee.19.8.5326

[14] A. R. Mehrabian, C. Lucas, "A novel numerical optimization algorithm inspired from weed colonization", Ecology Information, vol. 1, no. 4, pp. 355-366, 2006. [Online] Available: http://dx.doi.org/10.1016/j.ecoinf.2006.07.003

[15] S. Karimkashi, A. Kishk, "Invasive weed optimization and its features in electromagnetics", IEEE Trans. Antenna and Propagation, vol. 58, no. 4, pp. 1269-1278, 2010. [Online] Available: http://dx.doi.org/10.1109/TAP.2010.2041163

[16] M.C. Chang,W. C. Weng, "Synthesis of cosecant array factor pattern using particle swarm optimization", in Proc. International Symposium on Antennas \& Propagation (ISAP), Nanjing, 2013, vol. 2, pp. 948-951.

[17] G. G. Roy, S. Das, P. Chakraborty, P. N. Suganthan, "Design of nonuniform circular antenna arrays using a modified invasive weed optimization algorithm", IEEE Trans. Antennas And Propagation, vol. 59, no. 1, pp. 110-118, 2011. [Online] Available: http://dx.doi.org/10.1109/TAP.2010.2090477

[18] S. K. Goudos, V. Moysiadou, T. Samaras, K. Siakavara, J. N. Sahalos, "Application of a comprehensive learning particle swarm optimizer to unequally spaced linear array synthesis with side lobe level suppression and null control", IEEE antennas and wireless propagation letters, vol. 9, pp. 125-129, 2010. [Online] Available: http://dx.doi.org/10.1109/LAWP.2010.2044552 